\title{
As Equações de Hamilton sem Transformação de Legendre
}

Hamilton Equations dispensing with Legendre Transformation

\author{
G.F. Leal Ferreira \\ guilherm@if.sc.usp.br \\ Instituto de Física de São Carlos, USP \\ CP 369, 13560-970, São Carlos, SP
}

Recebido em 10 de janeiro de 2001. Aceito em 05 de fevereiro de 2001

\begin{abstract}
Maxwell, preparando sua abordagem dinâmica ao Eletromagnetismo (Treatise, Vol. II, Part IV, Cap.VI) alcança, no capítulo anterior, as equações de Hamilton pelo método das impulsões, que ele atribui a Thomson e Tait. O estado de movimento do sistema é dado em termos das coordenadas generalizadas e dos seus momentos, estes sendo vistos como resultado de impulsões convenientemente aplicadas a partir do repouso e da configuração atual do sistema. Esta abordagem é bem mais física do que a bem rápida baseada na transformação de Legendre e permite ver as equações de Hamilton como prescrevendo as mudanças temporais entre as grandezas de posição e de momento - entendidas como impulsos das forças aplicadas - e não como em Lagrange em que só grandezas associadas às massas, posição e velocidade, aparecem.
\end{abstract}

Maxwell, preparing his dynamical approach to the Electromagnetism (Treatise, Vol. II, Parte IV, Cap.VI), reaches in the previous chapter the Hamilton equations by the impulsive method, attributed by him to Thompson and Tait. The state of motion of the system is given in terms of the generalized coordinates and their momenta, these seen as a result of impulsive forces conveniently applied from rest and from the actual configuration of the system. This approach is much more physical than the one provided by the very rapid one through Legendre transformation and has the merit of allowing us to see the Hamilton equations as prescribing the mutual changes of positions and momenta - let them be understood as impulses of the applied forces - while in Lagrange's method deals only with quantities associated to the masses, position and velocities.

\section{Introdução}

A passagem da formulação de Lagrange à de Hamilton é, nos dias de hoje, realizada através de transformações de Legendre. Da lagrangeana, expressa em termos das posições $q_{i}$ e suas velocidades $\dot{q}_{i}$, constroi-se a função $H$, contendo a mesma informação, mas expressa em função dos $q_{i}$ e dos $p_{i}$, definidos como $\partial L / \partial \dot{q}_{i}$. É um caminho rápido, mas que dificilmente consegue dar conteudo físico aos momentos $p_{i}$ e às próprias equações de Hamilton que resultam da transformação. Maxwell, no seu Treatise [1], preparando sua Dynamical Theory of Electromagnetism, que aparece no capítulo $6^{\circ}$ da Parte IV do Vol. II, apresenta tratamento, que atribui a Thomson e Tait, em que a energia cinética, inicialmente expressa em termos dos $q_{i}$ e $\dot{q}_{i}$, é agora expressa em termos dos $q_{i}$ e $p_{i}$, estes sendo definidos como os momentos resultantes de impulsões realizadas a partir de cada configuração $q_{i}$, do repouso ao estado atual do sistema, com as velocidades $\dot{q}_{i}$. É o que reproduzimos a seguir, com poucas modificações e alguns comentários adicionais. De passagem mencionamos que em sua teoria dinâmica os circuitos elétricos em interação são considerados como um sistema descrito por coordenadas de posição e suas velocidades, enquanto as correntes são descritas por velocidades 'elétricas' cujas posições são ignoráveis.

\section{Os momentos como im- pulsões}

Sejam as equações de Lagrange para as coordenadas generalizadas $i$ do sistema

$$
\frac{d}{d t} \frac{\partial T_{L}}{\partial \dot{q}_{i}}-\frac{\partial T_{L}}{\partial q}=Q_{i}
$$

em que a energia cinética $T$, escrita como $T_{L}$, significa a expressão de $T$ em termos das variáveis lagrangeanas 
$q_{i}$ e $\dot{q}_{i} . Q_{i}$ é a força generalizada correspondente à coordenada $q_{i}$ e dada em termos das forças $\vec{F}_{j}$ aplicadas às $j$ partículas do sistema, com posição $\vec{r}_{j}$, como

$$
Q_{i}=\sum \vec{F}_{j} \cdot \frac{\partial \vec{r}_{j}}{\partial q_{i}}
$$

Quando for evidente a que índice o somatório se refere, ele não será exibido.

A energia cinética é, em geral, uma função quadrática das velocidades

$$
T_{L}=\frac{1}{2} \sum a_{i, j}(q) \dot{q}_{i} \dot{q}_{j},
$$

em que os coeficientes $a_{i, j}$ são funções das posições $q$ 's e satisfazem a relação $a_{i, j}(q)=a_{j, i}(q)$. Dada uma configuração do sistema, isto é, dadas as coordenadas $q_{i}$, qualquer estado de movimento do mesmo, isto é, qualquer conjunto de valores de velocidades $\dot{q}_{i}$, pode ser alcançado através de determinadas impulsões (impulsos instantâneos) [2]) $\vec{I}_{j}$ aplicadas às partículas a partir do repouso, naquela configuração. Os valores de $\dot{q}_{i}$ caracterizam, para cada configuração $q_{i}$, um conjunto de impulsões, que levariam o sistema do repouso àquele estado de movimento. Consideremos as impulsões $\vec{I}_{j}$ perpetradas no tempo $t$, ou melhor, entre $t_{-}$e $t_{+}$,

$$
\overrightarrow{I_{j}}=\int_{t_{-}}^{t_{+}} \vec{F}_{j} d t
$$

Integrando no tempo a Eq.(1) e tendo em conta a Eq.(4) temos

$$
\frac{\partial T_{L}}{\partial \dot{q}_{i}}=\sum_{j} \vec{I}_{j} \cdot \frac{\partial \vec{r}_{j}}{\partial q_{i}}+\sum_{j}\left(\frac{\partial a_{i, j}(q)}{\partial q_{i}}\right) \int_{t_{-}}^{t_{+}} \dot{q}_{i} \dot{q}_{j} d t
$$

Vê-se que a contribuição da integral se anula porque, por exemplo, $\dot{q}_{i} d t=d q_{i}=0$ e a variação máxima das velocidades, $\dot{q}_{j}$, é finita. Então, os $\partial T_{L} / \partial \dot{q}_{i}$ adquirem valores específicos $p_{i}$, os momentos, independentes agora da representação inicial lagrangeana. Temos então

$$
p_{i}=\sum \vec{I}_{j} \cdot \frac{\partial \vec{r}_{j}}{\partial q_{i}}=\sum a_{i, j}(q) \dot{q}_{j} .
$$

Para um dado estado do sistema, $q_{i}, \dot{q}_{i}$, o emprego do $2^{\mathrm{Q}}$ e do $3^{\mathrm{Q}}$ termo da igualdade acima poderia ser usado para se calcular um conjunto de impulsões que levaria o sistema às velocidades desejadas, mas não necessitamos delas para determinar os $p_{i}$, dados pelo $1^{\mathrm{O}}$ e $3^{\mathrm{O}}$ termo da Eq.(6). Portanto, o conjunto dos $p_{i}$ podem ser usados alternativamente aos $\dot{q}_{i}$ e a Eq.(6) indica como fazê-lo, isto é, invertendo-a com

$$
\dot{q}_{i}=\sum b_{i, j} p_{j} \quad \operatorname{com} \quad b_{i, j}=b_{j, i} .
$$

Embora as impulsões tenham sido usadas para caracterizar fisicamente os momentos $p_{i}$, e possam ser usadas livremente em deduções (veja, por exemplo, a obtenção da expressão da energia cinética na seção III), os momentos podem agora ser usados como variáveis contínuas no tempo. A substituição dos $\dot{q}_{i}$ pelos $p_{j}$ com a Eq.(7) na Eq.(3) forneceria a energia cinética $T_{H}$ em que ela é expressa em termos dos $q_{i}$ e $p_{i}$. Note-se que $T_{H}$, em vez de expressar-se só através de grandezas associadas às partículas $q_{i}$ e $\dot{q}_{i}$, usa as variáveis $p_{i}$ associadas às forças, ou aos seus impulsos e as equações de Hamilton vão dizer como se dão as mudanças recíprocas.

\section{Obtenção da relação $\partial T_{H} / \partial p_{i}=\dot{q}_{i}$, precursora de $\partial H / \partial p_{i}=\dot{q}_{i}$}

Com a representação $T_{H}$, estados com os mesmos $q_{i}$ mas com variados $p_{i}$ podem ser vistos como resultantes de aplicações de impulsões de magnitudes diferentes. Se a partir da mesma configuração dois conjuntos de impulsões, $p_{i}$ e $p_{i}+d p_{i}$, são aplicados a partir do repouso, a diferença de energia cinética alcançada é claramente dada por

$$
d T=\sum \frac{\partial T_{H}}{\partial p_{i}} d p_{i}
$$

já que as posições não mudam. Vamos mostrar que $d T$ também pode ser calculado como

$$
d T=\sum \dot{q}_{i} d p_{i}
$$

e, então, concluiremos das Eqs.(8) e (9) que $\dot{q}_{i}=$ $\partial T_{H} / \partial p_{i}$.

Para isto, vamos calcular primeiro a energia cinética comunicada nas impulsões que levam do repouso aos momentos $p_{i}$ através do trabalho $W$ realizado. Este vale

$W=\int_{t_{-}}^{t_{+}} \sum_{j} \vec{F}_{j} \cdot \sum_{i} \frac{\partial \vec{r}_{j}}{\partial q_{i}} d t=\int_{t_{-}}^{t_{+}} \sum_{j} \vec{F}_{j} \cdot \sum_{i} \frac{\partial \vec{r}_{j}}{\partial q_{i}} \dot{q}_{i} d t$

que podemos escrever como

$$
W=\sum_{i, j} \int_{t_{-}}^{t_{+}} \dot{q}_{i} \vec{F}_{j} \cdot \frac{\partial \vec{r}_{j}}{\partial q_{i}} d t=\sum_{i, j} \vec{I}_{j} \cdot \frac{\partial \vec{r}_{j}}{\partial q_{i}} \overline{\dot{q}}_{i}
$$

em que $\overline{\dot{q}}_{i}$ é a média das velocidades durante o impulso e que no caso presente é a metade da velocidade final $\dot{q}_{i}$. Pela Eq.(11) a energia cinética é então

$$
T=W=\frac{1}{2} \sum p_{i} \dot{q}_{i}
$$

expressão que, em vista das razões já apresentadas, é válida em geral, isto é, para qualquer tipo de movimento. Agora, das Eqs.(6) e (7), podemos concluir que, em vista da simetria dos coeficientes $a_{i, j}$ e $b_{i, j}$, temos

$$
\sum p_{i} d \dot{q}_{i}=\sum \dot{q}_{i} d p_{i}
$$


Retornando às impulsões $p_{i}$ e $p_{i}+d p_{i}$, nas quais as velocidades passam de $\dot{q}_{i}$ a $\dot{q}_{i}+d \dot{q}_{i}$, tira-se das Eqs.(12) e (13) que

$$
d T=\sum \dot{q}_{i} d p_{i}
$$

que comparada à Eq.(8) leva a

$$
\frac{\partial T_{H}}{\partial p_{i}}=\dot{q}_{i}
$$

que, praticamente, é uma das Equações de Hamilton.

\section{Obtenção da outra equação de Hamilton, $\partial H / \partial q_{i}=-\dot{p}_{i}$}

Seguindo [1], lembra-se primeiro que $d p=\dot{p} d t$ e que $d q=\dot{q} d t$ e usando a Eq.(15) temos que

$$
\frac{\partial T_{H}}{\partial p_{i}} d p_{i}=\dot{q}_{i} \dot{p}_{i} d t=\dot{p}_{i} d q_{i}
$$

Como

$$
d T=d T_{H}=\sum\left(\frac{\partial T_{H}}{\partial p_{i}} d p_{i}+\frac{\partial T_{H}}{\partial q_{i}} d q_{i}\right),
$$

segue da Eq.(16) que

$$
d T=d T_{H}=\sum\left(\dot{p}_{i}+\frac{\partial T_{H}}{\partial q_{i}}\right) d q_{i} .
$$

Supondo que o sistema seja conservativo e que, assim, o trabalho elementar das forças $d W_{c}$ se expressa através de uma energia potencial $U(q)$,

$$
d W_{c}=-\sum \frac{\partial U(q)}{\partial q_{i}} d q_{i}
$$

podemos igualar $d T$ a $d W_{c}$, Eq.(19), e obter

$$
d T_{H}=d T=d W_{c}=-\sum \frac{\partial U(q)}{\partial q_{i}} d q_{i}
$$

$$
=\sum\left(\dot{p}_{i}+\frac{\partial T_{H}}{\partial q_{i}}\right) d q_{i}
$$

e sendo os $d q_{i}$ independentes

$$
\frac{\partial H(q, p)}{\partial q_{i}}=\frac{\partial\left(T_{H}+U\right)}{\partial q_{i}}=-\dot{p}_{i}
$$

em que se definiu a hamiltoniana $H(q, p)$, reconhecida como a energia total do sistema. Como o potencial não depende das velocidades, a Eq.(15) pode ser reescrita como

$$
\frac{\partial H(q, p)}{\partial \dot{p}_{i}}=\dot{q}_{i}
$$

que é a outra equação de Hamilton.

\section{Conclusões}

Estamos agora convencidos de que na Mecânica de Hamilton, as forças, através de seus impulsos, tornam-se atores principais e não mero coadjuvantes como na de Lagrange. Sabemos também que o movimento contínuo de um sistema pode ser estudado como criado do repouso, na configuração atual, por impulsões, fornecendo um meio alternativo de abordagem.

\section{Agradecimentos}

O autor agradece a bolsa de produtividade ao $\mathrm{CNPq}$.

\section{References}

[1] J. Clerk Maxwell, A treatise on Electricity and Magnetism, Vol.2, Dover Public., Nova York, 1954, Parte IV, Cap. V.

[2] S. W. McCuskey, An Introduction to Advanced Dynamics, Addison-Wesley Publ. Co., Reading, 1959, Cap. 2. 